\title{
Radiation source deployment system for the calibration of the SuperNEMO detector
}

\author{
Ramon Salazar ${ }^{1}$ (on behalf of the SuperNEMO collaboration) \\ University of Texas at Austin \\ 2515 Speedway Stop C1600, Department of Physics, Austin, Texas, U.S. \\ E-mail: ramonmsalazar@gmail.com \\ Joshua Bryant \\ University of Texas at Austin \\ 2515 Speedway Stop C1600, Department of Physics, Austin, Texas, U.S. \\ E-mail: joshua.bryant.10@alumni.ucl.ac.uk
}

\begin{abstract}
An automatic source deployment system has been developed to introduce radioactive sources into the SuperNEMO detector for the purpose of calibration. Six plumb bobs are deployed from long wires which contain a number of well characterized sources at specific locations along their lengths. These sources are introduced using six stepper motors in a fully automated process which is both prompt and accurate. The position of each plumb bob is known using laser sensors which can stop the plumb bobs with a precision of $120 \mu \mathrm{m}$. Control and monitoring of the system is achieved using a National Instruments compactRIO which allows visual feedback through a graphical interface as well as remote access.
\end{abstract}

38th International Conference on High Energy Physics

3-10 August 2016

Chicago, USA

${ }^{1}$ Speaker 


\section{Introduction}

SuperNEMO is an experiment which is designed to search for the existence of neutrinoless double beta decay (Figure 1). Observations of this phenomenon would imply that neutrinos are Majorana particles. The goal of the experiment is to achieve a sensitivity to neutrinoless double beta decay corresponding to an effective mass of the order of $50 \mathrm{meV}$.

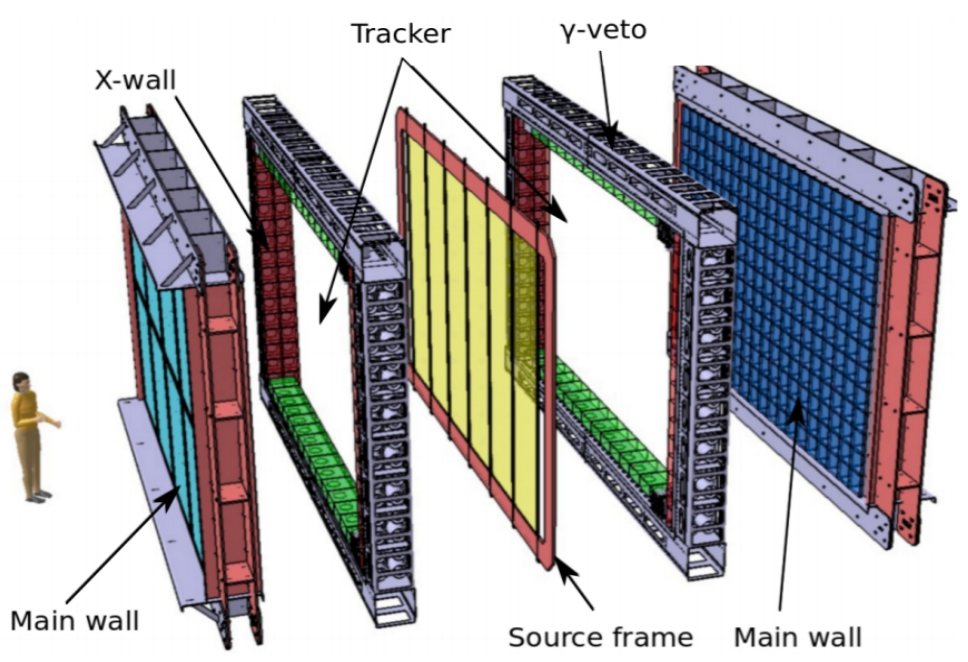

Figure 1. 3D Sketch of the SuperNEMO detector separated into its different sections [1].

\section{Calibration}

The experiment will collect double beta decay events by measuring electron energies in scintillator blocks read out by photomultipliers. This system is calibrated using radioactive sources, predominantly ${ }^{207} \mathrm{Bi}$, that are introduced into the detector through the automatic deployment calibration system.

\section{Deployment System}

The deployment system consists of six OFC plumb bobs suspended from stainless steel wires inside the SuperNEMO source frame (shown in Figure 1). Each wire is wrapped around a wheel on top of the detector which may be rotated by a stepper motor, lowering and raising each plumb bob. At three fixed points on each wire above the plumb bobs, ${ }^{207} \mathrm{Bi}$ calibration sources are attached, making it possible to introduce the sources into the detector.

At the bottom of the source frame there are six nests with laser light passing through them. Each plumb bob has a hole big enough for the laser beam to pass through. As a plumb bob enters a nest, it first interrupts the laser; this interruption is detected by a computer which slows down the motor. As the plumb bob continues descending, it reaches a position where its hole aligns with the laser beam (see Figure 2). The computer is once again alerted by this change, 
and it stops the motor completely. Six different plumb bobs enter the detector at different positions in the source frame.
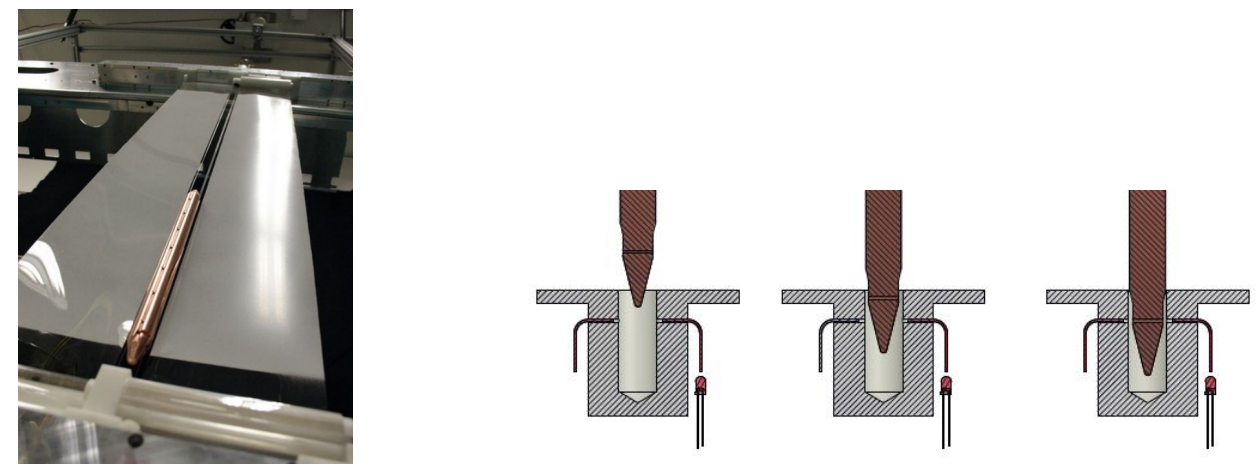

Figure 2. A sample plumb bob with two mock source foils beside it installed on a replica of the source frame (left). A plumb bob entering its nest, interrupting the laser light, and finally allowing the laser light to pass through the hole in its body (right)

\section{Automating the System}

To automate the system a photodiode amplifier is used to create electrical signals whenever it receives light from the laser. These signals are the way the computer knows when the laser is interrupted and uninterrupted. The computer, called CompactRIO, runs a program which communicates with the stepper motor drivers needed to control the stepper motors that deploy the plumb bobs. It has 64 inputs/outputs, and it is compatible with LabVIEW, the program used to control the motion.

\section{Operating the Valves}

Since the detector will be filled with a specific gaseous mixture $(94.85 \%$ helium, $4 \%$ ethyl alcohol, $1 \%$ argon, and $0.15 \%$ water), valves will be installed on the apertures which allow the plumb bobs to be introduced. They will be opened only during a calibration run. Once the run is over and the plumb bobs are extracted, the valves are closed again. These operations have also been automated using the same computer, but instead of using laser light to produce the electrical signals, mechanical switches are used (see Figure 3).

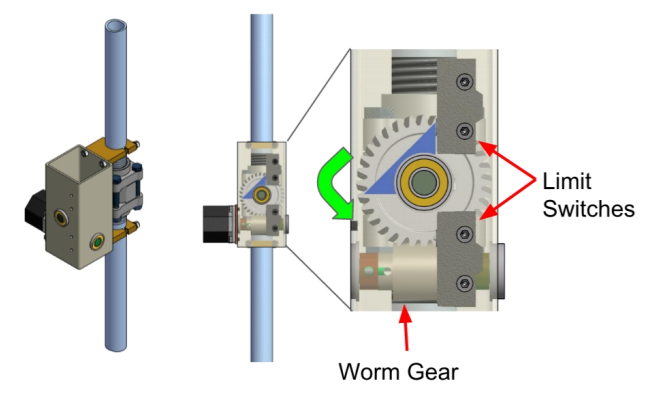

Figure 3. The mechanical design of the automatic valve system 


\section{Assembly}

Part of the system has been assembled (as shown in Figure 4), and it has been tested running four plumb bobs at a time for 42 hours straight. Each stepper motor requires one stepper motor driver. We need 12 of them (six for plumb bobs and six for valves). All of them have been connected to the CompactRIO successfully.
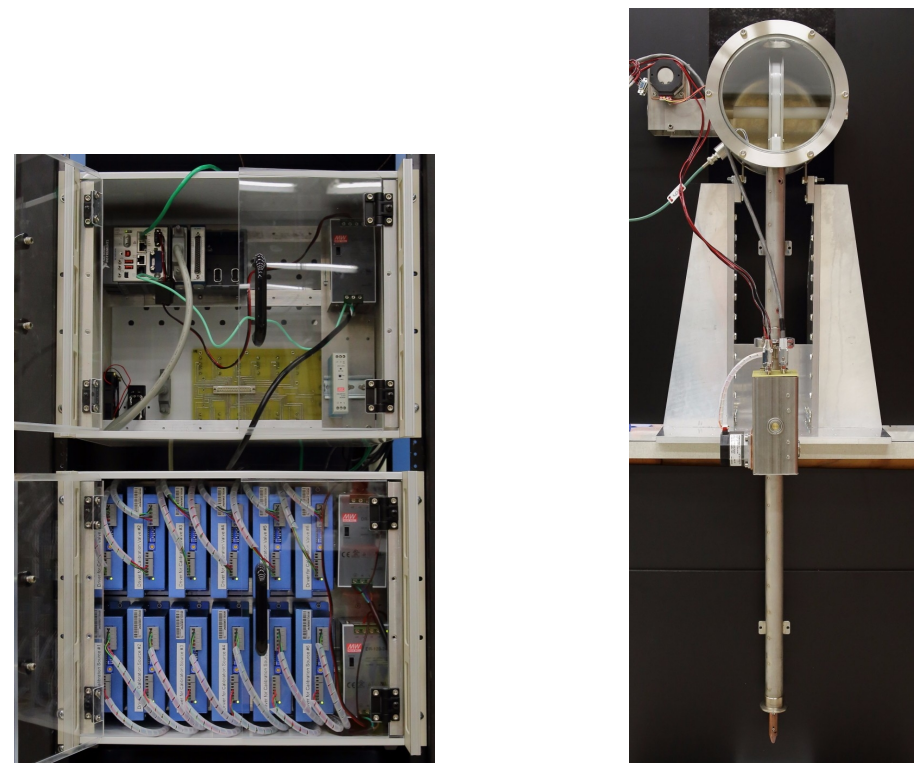

Figure 4. The electronics for the system are housed in two crates, the top one containing the compactRIO and the bottom one housing the 12 motor drivers (left). Each plumb bob enters the source frame through a metal pipe which can be automatically and hermetically sealed by closing the valve attached to the pipe (right).

\section{Conclusion}

An automatic source deployment calibration system has been designed for the SuperNEMO experiment. By introducing ${ }^{207} \mathrm{Bi}$ sources inside the source frame, it will become possible to convert the raw data collected into absolute energy measurements. The automation of the system will allow for easy, prompt, and precise calibrations.

\section{References}

[1] F. Perrot, Status of the SuperNEMO Demonstrator, $38^{\text {th }}$ International Conference on High Energy Physics (ICHEP 2016), Chicago, USA, August 3-10, 2016, PoS. 